\title{
Tatlockia micdadei
}

National Cancer Institute

\section{Source}

National Cancer Institute. Tatlockia micdadei. NCI Thesaurus. Code C86817.

A species of aerobic, Gram negative, rod shaped bacteria in the phylum Proteobacteria.

This species is motile, nonacid fast, nonencapsulated, nonfermentative, catalase positive.

T. micdadei is pathogenic only in immunocompromised patients, due to the decrease in virulence factors in relation to other microorganisms of this genus, being the causative agent of Pittsburgh pneumonia. 